
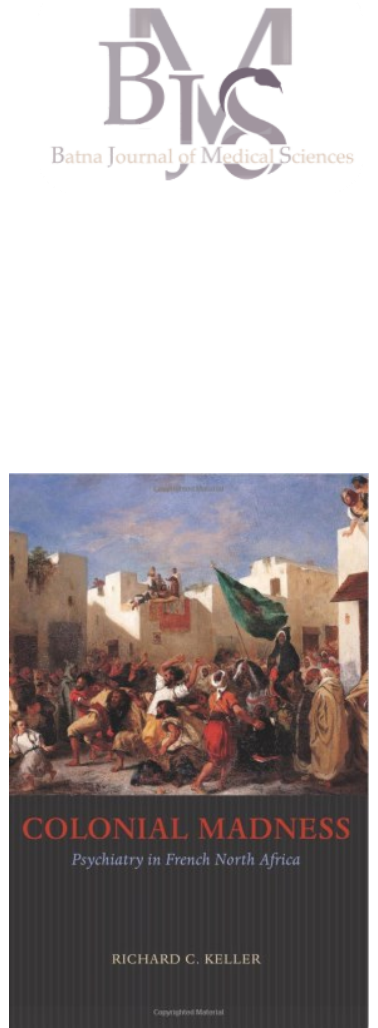

Correspondance à :

Zoubir BENMEBAREK

Service de psychiatrie, EHS psychiatrique El Madher, Batna - Algérie

zoubirbenmeb@gmail.com

DOI : $\underline{\text { https://doi.org/10.48087/ }}$ BJMSba.2015.2228

Il s'agit d'un article en libre accès distribué selon les termes de la licence Creative Commons Attribution International License (CC BY 4.0), qui autorise une utilisation, une distribution et une reproduction sans restriction sur tout support ou format, à condition que l'auteur original et la revue soient dûment crédités.

\section{Pour citer l'article :}

Benmebarek Z. Analyse de livre : « Colonial madness : psychiatry in French North Africa ». Richard C. Keller. Chicago: University of Chicago Press, 2007. 294 pp.Batna J Med Sci 2015;2(2):219-220. https://doi.org/10.48087/ BJMSba.2015.2228

\title{
"Colonial Madness : Psychiatry in French North Africa ». Richard C. Keller. Chicago : University of Chicago Press, 2007. 294 pp.
}

\author{
Analyse faite par : Dr. Zoubir Benmebarek
}

Richard C. Keller, professeur assistant en histoire médicale et en histoire de la science à l'université de Wisconsin-Madison, dresse dans son livre " histoire de la psychiatrie coloniale française en Afrique du Nord », la naissance et le développement de la psychiatrie coloniale française au Maghreb, les contours idéologiques qui ont émaillé son évolution et les particularités de sa pratique sur le terrain. Pour les besoins de la recherche sur le sujet, l'auteur s'est déplacé en France et en Tunisie mais paradoxalement pas en Algérie alors que le livre aurait pu être intitulé histoire de la psychiatrie française en Algérie tant ce pays est le centre d'intérêt du livre.

Le livre se situe en 06 chapitres couvrant la période du 19 ème siècle à l'indépendance des trois pays maghrébins.

Pinel au Maghreb est le premier chapitre du livre où l'auteur fait l'état des lieux de la psychiatrie et de ses structures existantes au 19 ème siècle. Ces structures datant de l'époque ottomane étaient archaïques et inadaptées (Maristan Tékia à Tunis et Seddiki à Rabat) et dont le rôle se limitait à enfermer et enchaîner les malades dangereux. Il décrit aussi la perception des médecins et aliénistes français à l'égard de la pathologie mentale au Maghreb marquées par un relent de colonialisme (le médecin promoteur de la civilisation française) et imprégnées des récits des orientalistes (l'aliénation source à la fois de peur et de fascination).

Durant cette période la solution pour la prise en charge des malades mentaux proposée par le régime colonial consistait en leur transfert vers des asiles du Sud de la France, pour y être internés parfois à vie loin de leurs familles et de leur patrie.

Dans le second chapitre l'auteur évoque la naissance difficile de la psychiatrie coloniale au Maghreb.

En 1911 la première clinique neuropsychiatrique est inaugurée à Tunis par Antoine Porot, psychiatre militaire qui devient ultérieurement chef de service de l'hôpital Maillot en 1916 et titulaire de la chaire de psychiatrie en Algérie en 1925. À l'issue du congrès des aliénistes français en 1912 s'est décidée l'ouverture de plusieurs hôpitaux psychiatriques en Algérie et au Maroc comme faisant partie de la mission civilisatrice de la France (hôpital Berrechid à Rabat, La Menouba à Tunis et Blida Joinville en Algérie). Il retrace les différentes étapes du déploiement et de l'organisation de la santé mentale et s'attarde sur les entraves liées à la construction de l'hôpital Blida Joinville (débat parmi les colons sur l'utilité de cet hôpital entre ceux qui y voient un devoir de la France et faisant partie de sa mission en Algérie et ceux, qui le considèrent comme une perte d'argent et une charge supplémentaire pour les contribuables).

Dans le troisième chapitre ayant pour titre «espaces d'expérimentation et sites de contestation », l'auteur décrit les difficultés et les particularités de la pratique psychiatrique en Algérie. Bénéficiant de conditions particulières et d'une large liberté de manœuvre comparées à leurs confrères en métropole, les psychiatres français avaient la chance de donner un essor remarquable à la psychiatrie coloniale en mettant en pratique des idées et des techniques difficilement acceptables en France (développement du système à deux lignes : les dispensaires comme première ligne de prise en charge et l'hôpital psychiatrique comme deuxième ligne, l'utilisation étendue des électrochocs, du choc insulinique et du cardiazol). Cet élan a été entravé par la surcharge des services d'hospitalisation par les malades chroniques venus de tous bords et par la barrière insurmontable colon-indigène.

Mais ce qui a vraiment marqué la psychiatrie coloniale et lui a fait rater un progrès indéniable c'est son racisme foncier et son essence colonialiste décortiquée au quatrième chapitre intitulé "entre clinique et savoir utile ».

Fondée en 1925 par Antoine Porot, l'école d'Alger s'est distinguée par une vision sectaire et raciste des musulmans. Mettant de côté le colonialisme, l'influence environnementale et la différence socio-culturelle et linguistique sur le comportement des algériens, elle a essayé moyennant des thèses pseudo scientifiques de coller une étiquette de " sous humains » aux indigènes avec des qualificatifs tels primitivisme, impulsivité innée, apathie, criminalité, crédulité...

Cette théorisation a donné crédit au colonialisme et a fait du savoir psychiatrique un alibi justifiant la violence coloniale à l'égard des autochtones. Ceci s'est poursuivi jusqu'à la guerre d'Algérie où l'armée a utilisé ce savoir 
(service d'action psychologique) dans sa lutte contre le FLN (Front de libération national).

Frantz Fanon, en poste à Blida en 1953, s'est attelé à déconstruire les thèses de l'école d'Alger en liant la maladie mentale au contexte particulier qu'est le colonialisme, générateur de troubles psychiques. En détruisant l'architecture sociale, marginalisant les autochtones, déniant leurs droits fondamentaux, il est à l'origine d'un malaise social profond et pourvoyeur d'un nombre croissant de maladies mentales.

Fanon en adaptant les techniques de psychothérapie institutionnelle, acquises à Saint-Alban, au contexte socio culturel algérien a eu un succès qui fût une preuve de l'inadéquation de l'approche de l'école psychiatrique d'Alger de la maladie mentale chez les algériens.

La psychiatrie est devenue, selon Fanon, un instrument de contrôle violent de la société en justifiant les pratiques inhumaines du colonialisme.

L'auteur reprend aussi dans ce chapitre les écrits de Kateb Yassine qui a fustigé l'ordre colonial à travers ses œuvres tel Nedjma, la femme sauvage, le cadavre encerclé etc. mais aussi à travers son histoire personnelle avec la maladie mentale de sa mère et son internement à Blida.

Le dernier chapitre est réservé à la période post-coloniale avec les conséquences de la colonisation sur le développement institutionnel au Maghreb mais aussi sur l'économie, la migration et l'échange culturel. Elle a été caractérisée par une insuffisance des ressources et un développement type asilaire des hôpitaux psychiatriques ne répondant pas aux besoins sociaux maghrébins, car étant une copie du système colonial et occidental.

À la fin du livre, l'auteur revient sur l'analyse de la migration maghrébine en France qui cristallise à un haut degré la relation colonisateur- colonisé et dévoile à travers la pathologie mentale des émigrés l'importance de la culture, de l'histoire et du déracinement dans l'expression de la maladie psychiatrique. Ceci a donné naissance à l'ethnopsychiatrie ou psychiatrie transculturelle (Georges Devereux, Tobie Nathan) qui essaye de contextualiser l'approche psychiatrique et éviter les préjugés et les stéréotypes qui ont longtemps caractérisé la vision occidentale de l'autre.

Le livre est parfois difficile à suivre car l'auteur a essayé d'embrasser toute l'étendue de la psychiatrie coloniale dans ses moindres détails, de ses fondements idéologiques aux résistances locales aux projets d'hôpitaux en passant par des histoires de patients, des extraits d'observations médicales, des débats politiques de l'époque ainsi que par la production littéraire et cinématographique liés de près ou de loin au sujet. Ceci dit, il demeure un éclairage riche, diversifié, indépendant et neutre sur le legs de la psychiatrie coloniale venant d'un auteur n'appartenant pas à l'ancienne puissance coloniale. Il ouvre le débat sur l'influence de l'histoire sur les perspectives de développement de l'institution psychiatrique en Algérie et au Maghreb et incite à la nécessité d'élaborer une vision nationale et autochtone sur ce parcours historique mais aussi sur son évolution actuelle. Sans cela il est illusoire de voir se développer une psychiatrie moderne et adaptée aux besoins de la population.

Cet article a été publié dans le « Batna Journal of Medical Sciences » BJMS, l'organe officiel de "I'association de la Recherche Pharmaceutique - Batna »

Le contenu de la Revue est ouvert « Open Access » et permet au lecteur de télécharger, d'utiliser le contenu dans un but personnel ou d'enseignement, sans demander l'autorisation de l'éditeur/auteur.

Avantages à publier dans BJMS :

- Open access : une fois publié, votre article est disponible gratuitement au téléchargement

- Soumission gratuite : pas de frais de soumission, contrairement à la plupart des revues « Open Access »

- Possibilité de publier dans 3 langues : français, anglais, arabe

- Qualité de la relecture : des relecteurs/reviewers indépendants géographiquement, respectant l'anonymat, pour garantir la neutralité et la qualité des manuscrits. 